\title{
The Utilization of Medical Devices by Traditional Korean Medicine Doctors Investigated through Traditional Korean Medicine Clinical Studies
}

\author{
Soo-Hyun Sung $\mathbb{D}^{1},{ }^{1}$ Hee-Ju Sim, ${ }^{2}$ Eu-Gene Kim, ${ }^{3}$ Angela Dongmin Sung $\mathbb{D},{ }^{4}$ \\ Jung-Youn Park, ${ }^{5}$ Byung-Cheul Shin $\mathbb{D}^{6},{ }^{6}$ Min-Jung Park $(\mathbb{D}){ }^{7}$ \\ Chang Hyun Han ${ }^{D},{ }^{8}$ and Jang-Kyung Park (iD) ${ }^{9}$ \\ ${ }^{1}$ Department of Pathology, College of Korean Medicine, Dae-gu Haany University, Daegu 38610, Republic of Korea \\ ${ }^{2}$ Department of Healthcare, Graduate School of Business, Ewha Womans University, Seoul 03760, Republic of Korea \\ ${ }^{3}$ Department of Food and Nutrition, College of Natural Sciences, Inha University, Incheon 22212, Republic of Korea \\ ${ }^{4}$ Department of Preventive Medicine, College of Korean Medicine, Sangii University, Wonju 26339, Republic of Korea \\ ${ }^{5}$ Department of Social Welfare, Sungkyunkwan University, Seoul 03063, Republic of Korea \\ ${ }^{6}$ Division of Clinical Medicine, School of Korean Medicine, Pusan National University, Yangsan 50612, Republic of Korea \\ ${ }^{7}$ Graduate School of Public Health, Seoul National University, Seoul 08826, Republic of Korea \\ ${ }^{8}$ Clinical Research Division, Korea Institute of Oriental Medicine, Daejeon 34054, Republic of Korea \\ ${ }^{9}$ Department of Obstetrics and Gynecology, College of Korean Medicine, Sangii University, Wonju 26339, Republic of Korea
}

Correspondence should be addressed to Jang-Kyung Park; vivat314@naver.com

Received 3 April 2018; Accepted 19 July 2018; Published 9 August 2018

Academic Editor: Kyung-Hwa Jung

Copyright (C) 2018 Soo-Hyun Sung et al. This is an open access article distributed under the Creative Commons Attribution License, which permits unrestricted use, distribution, and reproduction in any medium, provided the original work is properly cited.

\begin{abstract}
Objective. The purpose of this study was to investigate the current status of modern medical devices utilized in diagnosis and treatment in traditional Korean medicine (TKM). Methods. We searched the following six Korean electronic databases to collect TKM clinical studies that were published in a five-year period (January 2012 to December 2016). Clinical studies of TKM when medical devices were used for diagnosis or treatment were investigated. Results. The search generated a total of 3,735 articles, and 1,328 of these were considered to be clinical studies. Of a total of 1,328 clinical studies of TKM, 774 articles (58.3\%) used medical devices for diagnosis or treatment, and 554 articles (41.7\%) did not use medical devices for diagnosis or treatment. The three most used diagnostic devices were as follows: MRI scanners, which were used in 194 (20.6\%) studies; X-ray machines, which were used in 172 studies (18.3\%); and CT scanners, which were used in 139 studies (14.8\%). The three most used treatment devices were electroacupuncture equipment (20.3\%), transcutaneous electrical nerve stimulation (TENS) equipment (18.4\%), and interferential current therapy (ICT) equipment (16.4\%). Conclusions. This study suggests that TKM doctors use diagnostic information derived from modern medical devices clinically. It is therefore necessary to institutionalize considering changes to the medical acts of traditional medicine (TM) doctors. Additionally, this information can be utilized as a reference for developing TM policy and education.
\end{abstract}

\section{Introduction}

A medical device is an instrument, machine, device, material, or any other similar product used alone or in combination in humans or animals, as specified in the following: a product used for the purpose of diagnosing, curing, alleviating, treating, or preventing a disease; a product utilized for the purpose of diagnosing, curing, alleviating, or correcting an injury or impairment; a product used for testing, replacing, or transforming a structure or function [1]. According to the World Medical Market Report, the global medical device market will grow at an average annual rate of $4.8 \%$ from 2010 to 2014, rising to an annual average of $6.1 \%$ from 2015 to 2020 due to an increasing trend in aging and awareness [2]. It is estimated that the market size will grow to approximately $\$ 435.8$ billion by 2020 [2]. The size of the medical device 
market in Korea also maintained a high annual growth rate of $10.4 \%$ from 2011 to 2015 , and the production of medical devices reached $\$ 4.5$ billion in 2015 [3].

In the past, doctors showed the medical behaviors of using stethoscope; recently, they presented the behaviors of utilizing modern medical devices (e.g., magnetic resonance imaging (MRI) scanners, computed tomography (CT) scanners, and medical laser) for diagnosing and treating patients $[4,5]$. The reason why doctors have adopted modern medical devices is to gain a technical advantage and enhance selfdetermination and professional recognition [6].

Traditional tools such as acupuncture, herbal medicine, and cupping therapy have been used by traditional Korean medicine (TKM) doctors for a long time [7, 8]. Recently, TKM doctors have used medical devices such as electroacupuncture devices, electropulse graph, and distal arterial pulse wave analyzers, in combination with modern technology, for treatment $[9,10]$. However, certain medical devices such as X-ray machines, MRI scanners, and CT scanners are not legalized for use by TKM doctors $[11,12]$.

China and Taiwan, similar to Korea, have a dual medical system that combines traditional medicine (TM) with conventional medicine (CM), and TM is included in the national system to provide medical service for nations [13]. In China, traditional Chinese medicine (TCM) doctors prescribe conventional medications, use modern medical devices, and perform surgeries, similar to licensed medical doctors $[7,14]$. In Taiwan, only traditional treatments (e.g., acupuncture, moxibustion, and herbal medicine) were available to TM doctors, but recently complete blood counts, urinalysis, fecal analysis, and radiography have been allowed $[7,15]$. Thus, the practice of TM doctors varies according to national systems and policies.

To our knowledge, the review on the use of medical devices by TKM doctors has not been published. The aim of this study was to investigate the current status of the medical behavior of TKM doctors by analyzing the data from clinical studies of TKM journals and to provide a reference for establishing TM policies and systems in countries that have a TM system

\section{Methods}

2.1. Data Sources and Searches. In April 2017, we searched the following six Korean electronic databases to collect TKM clinical studies that were published recent five years (January 2012 to December 2016); Korea Institute of Science and Technology Information, Korean traditional knowledge portal, KoreaMed, OASIS, RISS, and KISS. Clinical trials of TKM indexed in non-Korea databases such as PUBMED, EMBASE, or MEDLINE were not considered. The search keywords were as follows: "oriental medicine OR traditional medicine OR traditional Korean medicine OR complementary and alternative medicine" AND "clinical studies OR clinical trial OR case studies OR case report OR case series OR case controlled trial OR randomized controlled trial".

We did not limit study languages, but we excluded studies that did not have a paper format (e.g., abstract-only articles and conference presentations). The studies of master and doctoral degrees were not considered for inclusion.

2.2. Study Selection. We included only clinical studies of TKM. Reviews, surveys, qualitative studies, and experimental studies were excluded. Cohort studies or clinical studies in which participants were not provided any type of treatment were also excluded. Three authors (H. J. Sim, E. G. Kim, and A. D. Sung) independently screened and selected papers that met the study criteria. Disagreements were resolved by discussion with two authors (S. H. Sung and J. K. Park) to arrive at a consensus

2.3. Definition of Medical Device. This study classified medical devices into two categories: diagnostic devices and treatment devices. Diagnostic devices include medical imaging equipment (e.g., X-ray machines, MRI scanners, CT scanners, and ultrasound scanners), blood testing equipment, and infrared thermographic equipment used to examine the human body. Treatment devices include equipment for electric needle treatment, equipment for electric stimulus treatment, and laser treatment devices. However, equipment such as needles, lancets, and cups for cupping were excluded from the study as they were not considered to be machines.

2.4. Data Extraction. In TKM clinical studies where medical devices were used for treatment or diagnosis, we gathered information regarding the medical device. The collected information was classified into diagnostic devices and treatment devices. Furthermore, in order to investigate the current status of medical device use per year and the type of diseases, relevant information was gathered and further analyzed. Two independent reviewers (J. Y. Park and C. H. Han) extracted data using a form designed in the review. Discrepancies were resolved by discussions with two other reviewers (S. H. Sung and B. C. Shin).

When there were multiple diseases, the more serious disease was put first, and studies that focused on healthy people or case studies that treated more than 2 people with multiple diseases were excluded.

\section{Results}

3.1. Study Selection and Description. The search generated a total of 3,735 articles, of which 1,328 were considered to be TKM clinical studies. Four hundred and thirty-six studies were excluded for the following reasons: 117 studies were reviews, seven studies were surveys, 71 studies were experimental studies, 238 studies were clinical studies without interventions, and three studies were abstract-only articles or conference presentations (Figure 1).

\subsection{Utilization of Medical Devices Classified by Type}

3.2.1. Utilization of Medical Devices. From a total of 1,328 clinical studies of TKM, 774 articles (58.3\%) used medical devices for diagnosis or treatment, and 554 articles (41.7\%) did not use medical devices for diagnosis or treatment. 


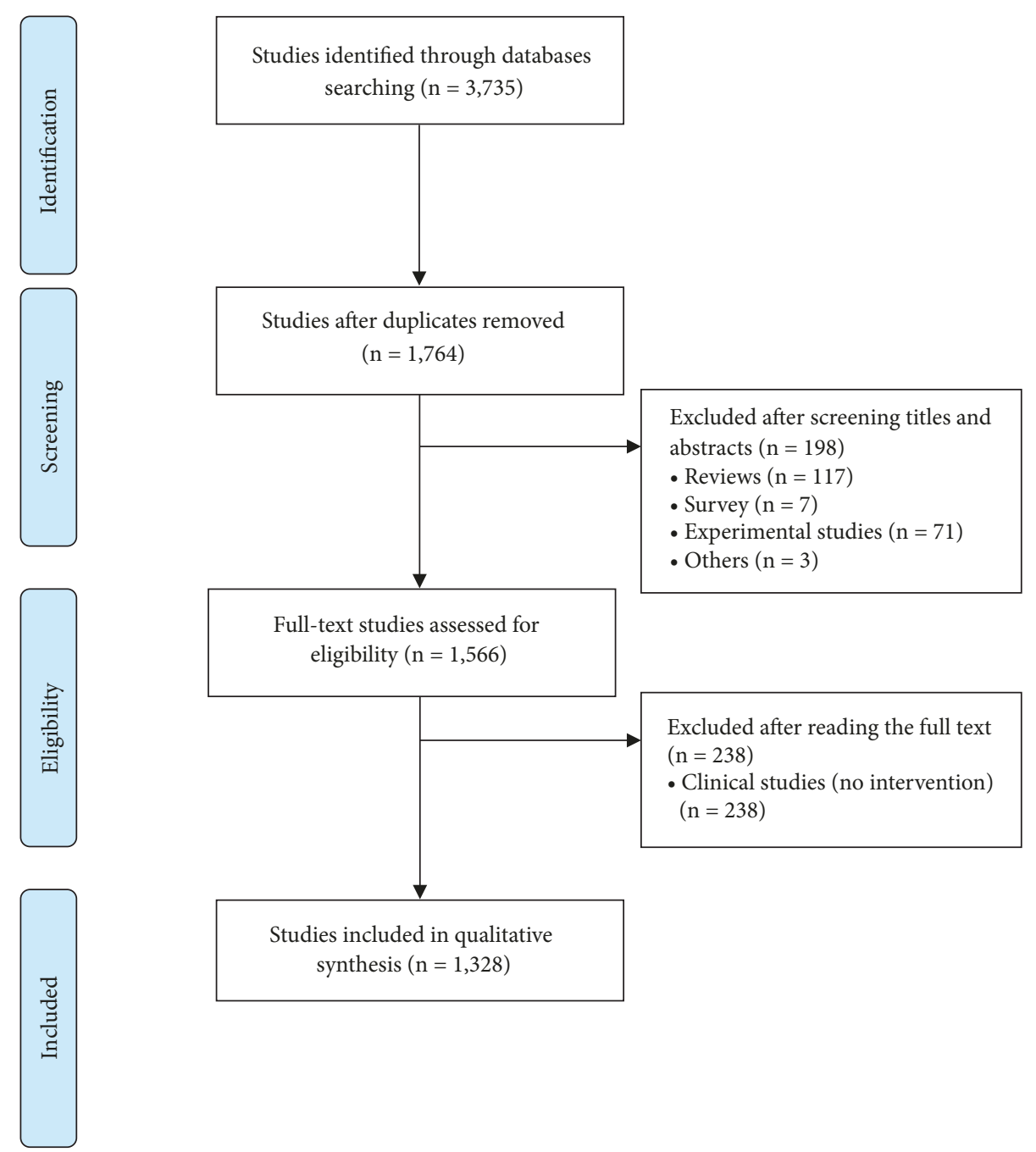

FIGURE 1: Flowchart of the study selection process.

Medical devices were used in TKM clinical studies a total of 1,345 times from 2012 to 2016; diagnostic devices were used 942 times (70\%), while treatment devices were used 403 times (30\%) (Table 1).

3.2.2. Utilization of Diagnostic Devices. Diagnostic devices were used 942 times in the TKM clinical studies. The 10 most used diagnostic devices are illustrated in Table 1. MRI scanners were used in 194 (20.6\%) studies; X-ray machines were used in 172 studies (18.3\%); CT scanners were used in 139 studies (14.8\%); electrocardiography (EKG) equipment was used in 80 studies $(8.5 \%)$; ultrasound scanners were used in 57 studies (6.1\%); digital infrared thermal imaging (DITI) equipment was used in 43 studies (4.6\%); heart rate variability (HRV) measurement equipment was used in 41 studies (4.4\%); body composition analyzers were used in 28 studies (3.0\%); electromyography (EMG) equipment was used in 21 studies (2.2\%); and endoscopes in 19 studies $(2.0 \%)$.
3.2.3. Utilization of Treatment Devices. In the 1,328 clinical studies investigated, medical devices were used for treatment 403 times. The 10 most used treatment devices are listed in Table 1. Electroacupuncture devices were used in 82 studies (20.3\%); transcutaneous electrical nerve stimulation (TENS) equipment was used in 74 studies (18.4\%); interferential current therapy (ICT) equipment was used in 66 studies $(16.4 \%)$; ultrasound and infrared (IR) devices were used in 23 studies (5.7\%); microwave therapy (M/W) equipment were used in 19 studies (4.7\%); electrical stimulation treatment (EST) equipment was used in 12 studies (3.0\%); lasers and silver spike points (SSPs) were used in 11 studies (2.7\%); and electromagnetic stimulation therapy equipment was used in 6 studies $(1.5 \%)$.

\subsection{Utilization of Medical Devices per Year}

3.3.1. Utilization of Medical Devices per Year. The use of medical devices decreased from 2012 to 2014 and increased by 
TABLE 1: Medical devices reported in TKM clinical studies.

\begin{tabular}{|c|c|c|}
\hline Types of Medical Device & Top 10 used Medical Device & $\begin{array}{c}\text { Number of papers } \\
\mathrm{n}(\%)\end{array}$ \\
\hline \multirow{11}{*}{ Diagnostic device 942} & MRI scanners & $194(20.6)$ \\
\hline & $\mathrm{X}$-ray machines & $172(18.3)$ \\
\hline & CT scanners & $139(14.8)$ \\
\hline & EKG equipment & $80(8.5)$ \\
\hline & Ultrasound scanners & $57(6.1)$ \\
\hline & DITI equipment & $43(4.6)$ \\
\hline & HRV measurement equipment & $41(4.4)$ \\
\hline & Body composition analyzer & $28(3.0)$ \\
\hline & EMG equipment & $21(2.2)$ \\
\hline & Endoscope & $19(2.0)$ \\
\hline & Etc. & 148 \\
\hline \multirow{11}{*}{ Treatment device 403} & Electro acupuncture devices & $82(20.3)$ \\
\hline & TENS equipment & $74(18.4)$ \\
\hline & ICT equipment & $66(16.4)$ \\
\hline & Ultrasound devices & $23(5.7)$ \\
\hline & IR equipment & $23(5.7)$ \\
\hline & $\mathrm{M} / \mathrm{W}$ equipment & $19(4.7)$ \\
\hline & EST equipment & $12(3.0)$ \\
\hline & Laser devices & $11(2.7)$ \\
\hline & SSP equipment & $11(2.7)$ \\
\hline & $\begin{array}{l}\text { Electromagnetic stimulation } \\
\text { therapy equipment }\end{array}$ & $6(1.5)$ \\
\hline & Etc. & 76 \\
\hline
\end{tabular}

MRI: magnetic resonance imaging; CT: computed tomography; EKG: electrocardiography; DITI: digital infrared thermal imaging; HRV: heart rate variability; EMG: electromyography; TENS: transcutaneous electrical nerve stimulation; ICT: interferential current therapy; IR: infrared rays; M/W: microwave therapy; EST: electrical stimulation therapy; SSP: silver spike point.

2016. Medical devices were used the most (312 times) in 2012. The utilization of diagnostic devices revealed a decreasing trend overall. The use of treatment devices declined from 2012 to 2015, and a steep increase occurred in 2016 (Figure 2).

3.3.2. Utilization of Diagnostic Devices per Year. Investigation of the 10 most used diagnostic medical devices per year in TKM clinical studies over the past 5 years revealed that MRI scanners, X-ray machines, and CT scanners were the most used medical devices from 2012 to 2016. MRI scanners were used a minimum of 32 and a maximum of 46 times every year. X-ray machines were used from 19 to 40 times, and CT scanners were used from 16 to 40 times. Electrocardiography use showed a decreasing trend from 2012. However, an increasing trend was demonstrated from 2014. Heart rate variability machines were not used much in 2015 and 2016, whereas they were used in 22 studies in 2012 (Figure 3).

3.3.3. Utilization of Treatment Devices per Year. Assessment of the 10 most used treatment devices that were utilized in more than 10 clinical studies per year revealed that electroacupuncture, TENS, and ICT devices were used on a regular basis every year, and IR equipment, laser equipment, and SSPs were used frequently in 2016 (Figure 4).

\subsection{Diagnosis and Treatment of Diseases per Type of Medical Device}

3.4.1. Diseases Diagnosed by Diagnostic Devices. The 5 most used diagnostic medical devices were used for various diseases. MRI was used to diagnose herniation of the lumbar disc in 16 studies (8.2\%), stroke in 12 studies (6.2\%), and rotator cuff tear in 5 studies (2.6\%). X-ray machines were used for diagnosing scoliosis in 9 studies (5.2\%) and pneumonia and herniation of the lumbar disc in 4 studies (2.3\%). CT scanners were used to diagnose stroke in 8 studies $(5.8 \%)$, lung cancer in 5 studies (3.6\%), and herniation of the lumbar disc and gastric cancer in 4 studies (2.9\%). For diagnosing amyotrophic lateral sclerosis and stroke, EKG was utilized in 4 studies (5.0\%). Ultrasound devices were utilized in diagnosing infertility in 4 studies (7.0\%), uterine myoma in 3 studies (5.3\%), and hepatitis, frozen shoulders, gastrocnemius tear, drug-induced liver injuries, and uterine bleeding in 2 studies (3.5\%) (Table 2). 


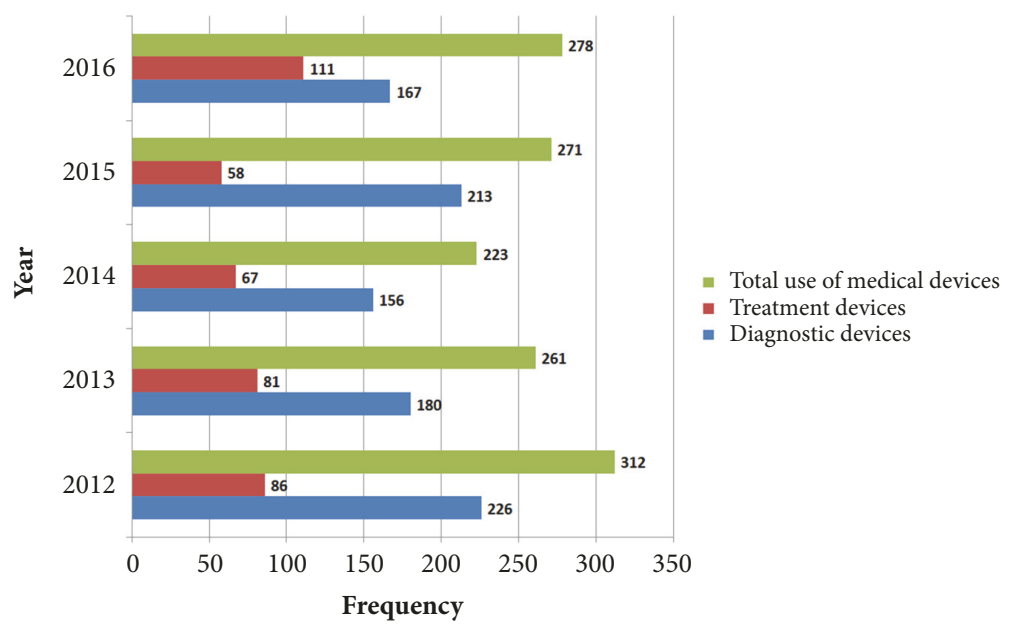

FIGURE 2: Utilization of medical devices in Korea from 2012 to 2016 by publication year.

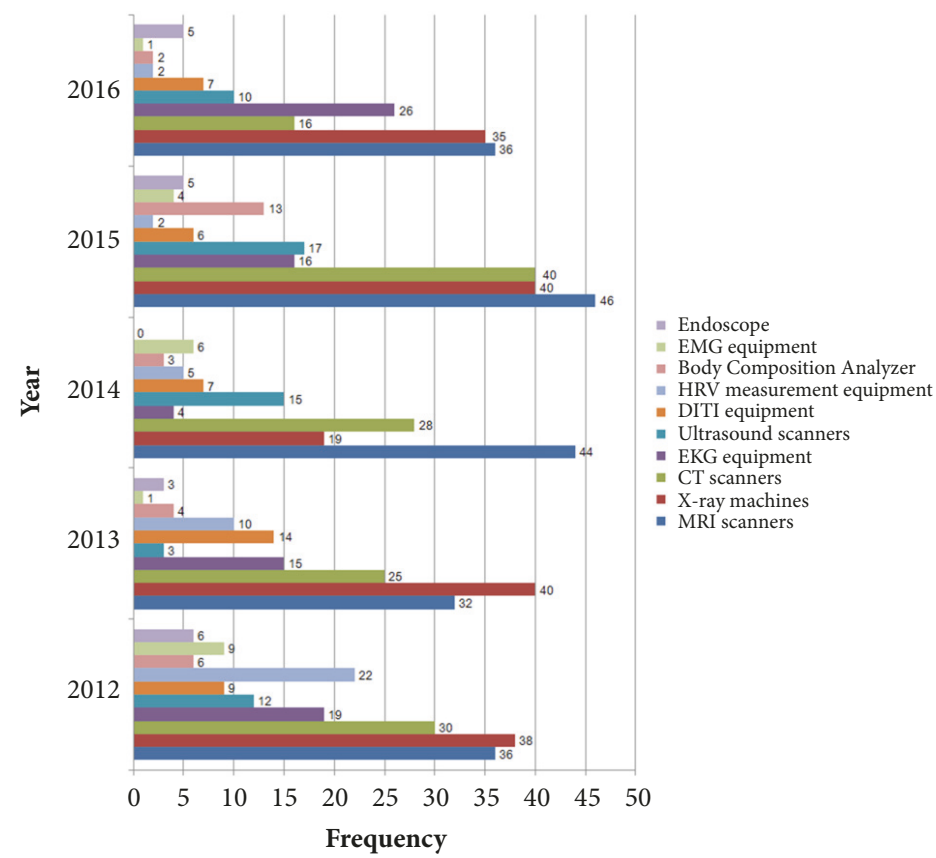

FIGURE 3: Study trend of diagnostic devices reported in clinical studies of TKM per year. MRI: magnetic resonance imaging; CT: computed tomography; EKG: electrocardiography; DITI: digital infrared thermal imaging; HRV: heart rate variability; EMG: electromyography.

3.4.2. Diseases Treated by Medical Treatment Devices. Investigation of the diseases treated by the 5 most used medical treatment devices revealed that electroacupuncture was used in treating facial palsy in 9 studies (11.0\%), stroke in 5 studies (6.1\%), and obesity and headaches in 3 studies (4.1\%). Transcutaneous electrical nerve stimulation was utilized in treating herniation of the lumbar disc in 11 studies (14.9\%), back pain in 4 studies (5.4\%), and shoulder pain in 3 studies (4.1\%). Interferential current therapy was used for treating herniation of the lumbar disc, in 7 studies (10.6\%), while ultrasonography was used in 3 studies (13.0\%). Infrared devices were used in 5 different studies $(21.7 \%)$ to treat facial palsy (Table 3).

\section{Discussion}

The medical devices are important for quality of health service delivery [16]. It is an applied technique that combines various subject fields such as clinical medicine, electricity, electronics, mechanics, and optical science [17]. The industry is part of the health and medical treatment industry, with the aim of improving the quality of life of humans. The current medical device industry in Korea has gone beyond the simple research stage and is transforming itself to a customized industry where replaceable biomaterial and artificial organs are being developed with high technology [18].

When investigating previous research regarding TKM doctors use of medical devices, Kim et al. [19] conducted 


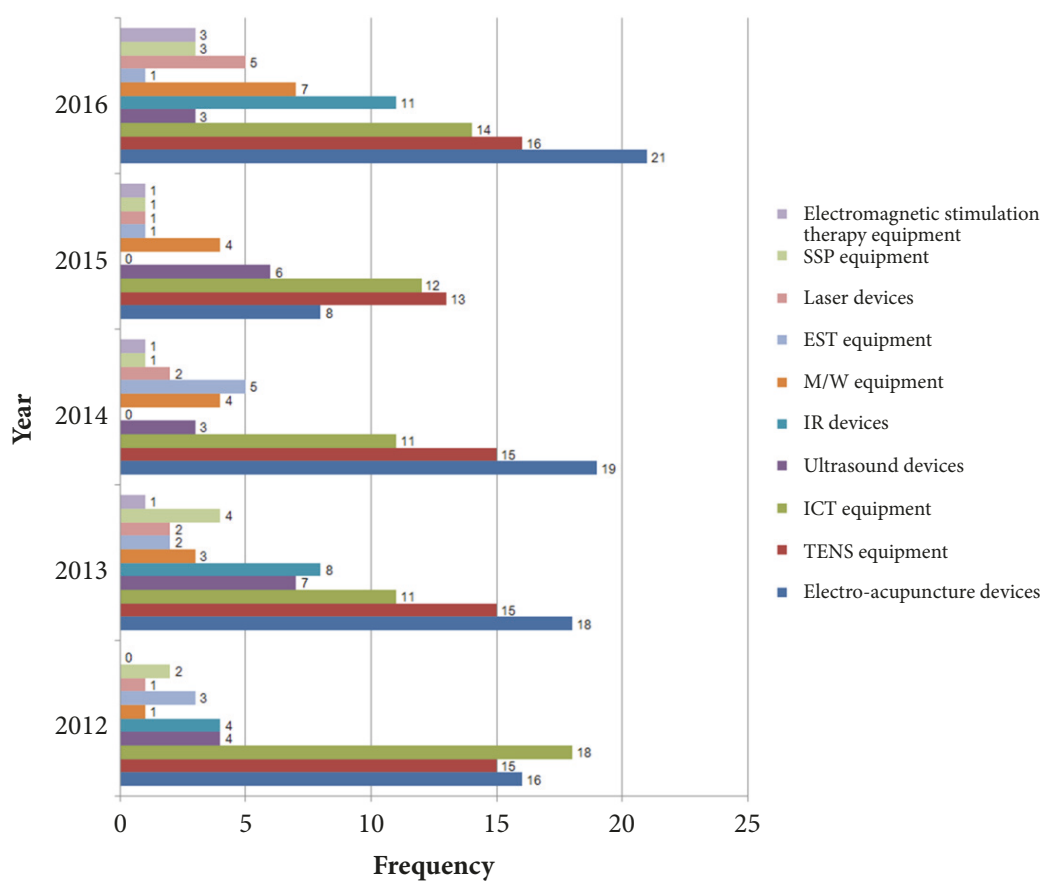

FIGURE 4: Study trend of treatment devices reported in clinical studies of TKM per year. TENS: transcutaneous electrical nerve stimulation; ICT: interferential current therapy; IR: infrared rays; M/W: microwave therapy; EST: electrical stimulation therapy; SSP: silver spike point.

TABLE 2: List of diseases diagnosed by diagnostic devices.

\begin{tabular}{|c|c|c|}
\hline Diagnostic Devices & Diseases of Top 3 used & $\mathrm{n}(\%)$ \\
\hline \multirow{3}{*}{ MRI scanners } & Herniation of lumbar disc & $16(8.2)$ \\
\hline & Stroke & $12(6.2)$ \\
\hline & Rotator cuff tear & $5(2.6)$ \\
\hline \multirow{2}{*}{ X-ray machines } & Scoliosis & $9(5.2)$ \\
\hline & Pneumonia and herniation of lumbar disc & $4(2.3)$ \\
\hline \multirow{3}{*}{ CT scanners } & Stroke & $8(5.8)$ \\
\hline & Lung cancer & $5(3.6)$ \\
\hline & Herniation of lumbar disc and gastric cancer & $4(2.9)$ \\
\hline \multirow{2}{*}{ EKG equipment } & Amyotrophic lateral sclerosis and stroke & $4(5.0)$ \\
\hline & Parkinson disease & $2(2.5)$ \\
\hline \multirow{3}{*}{ Ultrasound scanners } & Infertility & $4(7.0)$ \\
\hline & Uterine myoma & $3(5.3)$ \\
\hline & Hepatitis, frozen sholder, gastrocnemius tear, drug-induced liver injury and uterine bleeding & $2(3.5)$ \\
\hline
\end{tabular}

MRI: magnetic resonance imaging; CT: computed tomography; EKG: electrocardiography.

a survey where 900 TKM doctors were asked what types of medical devices are kept in TKM institutions. It was stated that although medical imaging equipment such as $\mathrm{X}$-ray machines, CT scanners, and MRI scanners were not kept as much in these institutions, the frequency of their use was high. Sakong et al's [20] research provided critical views on restrictions imposed on TKM doctors in using medical imaging equipment by investigating cases where TKM doctors were restricted from using medical imaging equipment, how medical imaging equipment was brought into the medical industry, and the academic principles and study curriculum of TKM.
In Taiwan, there are no laws prohibiting the use of modern medical devices by TCM doctors, and some medical devices are available for the ministry of health $[7,15]$.

In China, there are no laws prohibiting the use of modern medical devices by TCM doctors, and the government does not restrict the use of modern medical devices and western medicine prescriptions by the Chinese government [7, 21]. For this reason, many studies have been conducted combining TCM with medical imaging devices [22-24].

This study shows that diagnostic devices have been used more than treatment devices in TKM clinical studies. When investigating the diagnostic medical devices and treatment 
TABLE 3: List of diseases treated by treatment devices.

\begin{tabular}{lcc}
\hline Treatment Devices & Diseases of Top 3 used & $\mathrm{n}(\%)$ \\
\hline Electro acupuncture & Facial paralysis & $9(11.0)$ \\
& Stroke & $3(6.1)$ \\
& Obesity and headache & $13(14.9)$ \\
TENS equipment & Herniation of lumbar disc & $4(5.4)$ \\
& Back pain & $3(4.1)$ \\
\hline & Shoulder pain & $7(10.6)$ \\
ICT equipment & Herniation of lumbar disc & $5(7.6)$ \\
& Back pain & $4(6.1)$ \\
\hline Ultrasound devices & Neck pain & $3(13.0)$ \\
\hline IR devices & Herniation of lumbar disc & $5(21.7)$ \\
\hline
\end{tabular}

TENS: transcutaneous nerve stimulation; ICT: interferential current therapy; IR: infrared rays.

devices used in the TKM clinical studies, the statistics of the usage per year show that both types of devices have been used steadily from 2012 to 2016. In the past, TKM doctors made diagnosis based on the traditional observing, smelling, asking, and touching methods and treated their patients with acupuncture, moxibustion, and herbal medicine. Currently, medical devices are used for diagnosis and treatment, indicating advancement in treatment. Initially, even doctors did not use medical devices for diagnosing and treating patients. Based on medical knowledge, objective information driven from utilizing modern medical devices allowed for this change, and it is thought that these types of medical acts will further transform themselves with the development of scientific technology.

The use of MRI scanners, X-ray machines, and CT scanners accounted for $53.7 \%$ of the total diagnostic device usage from 2012 to 2016 in TKM clinical studies. However, MRI scanners, CT scanners, X-ray machines, and diagnostic ultrasound machines are medical devices that TKM doctors are not allowed to use by law [25-27], and it is estimated that TKM institutions gather and utilize health information such as image materials taken from CM hospitals or image materials gathered through joint treatment of both TKM and CM doctors.

Clauses 37 and 38 under the Medical Service Act state each of the installment regulations and managing director decisions for MRI, radiography, and CT, and TKM doctors do not have the authority to direct [28]. Clause 1 of the Medical Technicians Act states that leadership over medical technicians can be given to dentists or medical doctors [29]. Furthermore, most of the preceding cases state that the use of modern diagnostic devices by TKM doctors is not permitted. However, there was an exceptional case where the use of 5 different medical devices (e.g., applanation tonometer, autorefractor and keratometer, slit lamp, pure tone audiometer, and automated perimeter) were allowed to a TKM doctor at the constitutional court [30]. The reasons for the denial are thought to be a dual medical system, differences in academic principles, insufficient legal grounds for TKM doctors using medical devices, and insufficient educational institutions. This study suggests that TKM doctors use diagnostic information derived from modern medical devices clinically. In order for the TKM study, which is heavily based on traditional knowledge and experience, to go through the process of digitization and produce evidence, collecting objective information by utilizing medical devices is needed. Although there can be differences in the methods of treatment in TKM and CM, the right to use medical devices utilized for treatment and diagnosing patients should be equally given to both types of doctors. Interferential current therapy and TENS are used for treatment every year. It is thought that they have the effect of relieving muscle tension, making the flow of qi smooth, and stimulating the meridian point to improve the pain. The use of the same treatment device may vary depending on the type of medical occupation. It is necessary to consider this aspect when developing medical devices and licensing items.

Diagnostic medical devices such as MRI scanners, CT scanners, and X-ray machines were widely used in herniation of the lumbar disc. In Korea, the Ministry of Health and Welfare operates a system to designate specialized hospitals that treat specific diseases when the medical institute meets the standards set by Medical Law [31]. A total of 109 specialized hospitals have been designated as of 2018; in the TKM field, 8 TKM hospitals specialized in the spine and 1 TKM hospital specialized in gynecology were designated [31]. It is thought that the results of this study reflect that MRI scanners, CT scanners, and X-ray machines are used for treatment in hospitals that specialize in the spine. Conventional medicine and TKM doctors in these hospitals cooperate in areas of treatment-related research [32].

There are some limitations in this review. First, TKM clinical studies indexed in English databases, including EMBASE and MEDLINE, were not considered in our review. Therefore, it is difficult to see that the clinical trials studies related to TKM included in this paper are representative of the use of medical devices in TKM doctors.

Second, it was impossible to fully explore how TKM doctors utilize medical devices as they have restrictions in using these devices in their practice. To understand how these medical devices are used in actual practice, studies such as surveys or interviews are needed and investigations of 
medical behaviors of TM doctors in China and Taiwan are required.

Third, most of the clinical studies included in this review were conducted in TKM hospitals, and TKM hospitals make up only $1.9 \%$ of the TKM institutions [33]. It is therefore difficult to conclude that this review represents the majority of practices being carried out in TKM institutions.

Although TKM has been responsible for Korean people's health for thousands of years and holding sufficient historical evidence, there is no modern scientific evidence to support TKM. Therefore, this review is valuable because the medical behavior of TKM doctors was investigated for the first time. We cautiously propose that this review contributes to establishing TM policies in countries that practice TM and providing improved medical services of TM to patients.

\section{Conclusion}

We reviewed the use of medical devices by TKM doctors in Korea. It provided an understanding about the recent status and trends of the medical behaviors of TKM doctors related to medical devices. This study suggests that TKM doctors utilize medical devices for diagnosis and treatment of patients. It is necessary to institutionalize considering making changes to the medical acts of TM doctors. Additionally, this information can be utilized as a reference for making TM policy and education.

\section{Conflicts of Interest}

The authors declare that there are no conflicts of interest regarding the publication of this paper.

\section{References}

[1] National Law Information Center, Medical Device Act, http://www.law.go.kr/\%EB\%B2\%95\%EB\%A0\%B9/\%EC\%9D\% 98\%EB\%A3\%8C\%EA \%B8\%B0\%EA\%B8\%B0\%EB\%B2\%95.

[2] BMI Espicom, The World Medical Markets Factbook 2015, Epicom Business Intelligence Ltd, Chichester, UK, 2015.

[3] Korea Health Industry Development Institute, Analysis Report of Medical Device Industry, Korea Health Industry Development Institute, Hanhagmunhwa, Republic of Korea, 2016.

[4] Naver Encyclopedia, Stethoscope, 2017, http://terms.naver.com/ entry.nhn?docId=2843017\&amp;cid=55589\&amp; categoryId= 55589.

[5] M. Ghahramanifar, M. Haghani, A. Ghadimi Moghadam, and A. K. Ghadimi Moghadam, "A new stethoscope design with unique characteristics and development in medical device," Journal of Biomedical Physics and Engineering, vol. 8, no. 1, pp. 147-150, 2018.

[6] M. H. M. Hatz, T. Sonnenschein, and C. R. Blankart, "The PMA Scale: A Measure of Physicians' Motivation to Adopt Medical Devices," Value in Health, vol. 20, no. 4, pp. 533-541, 2017.

[7] K. J. Yoon and D. S. Kim, "Medical Unification Plan for shared growth with modern medicine and traditional medicine," pp. 27-116, Korea Institute for Health and Social Affairs, Sejong, Republic of Korea, 2013.

[8] Gihyun Lee, Wonnam Kim, Woojin Kim, and Hanbing Li, "Modernization of Traditional Oriental Medicine: New Dosage
Forms and Medical Instruments," Evidence-Based Complementary and Alternative Medicine, vol. 2018, Article ID 6960125, 1 page, 2018.

[9] E. S. Kim, C. G. Park, and J. G. Son, "Diagnostic Device of Traditional Korean Medicine," pp. 5-14, Korea Institute of Science and Technology Information, Seoul, Republic of Korea, 2004.

[10] Gihyun Lee and Woojin Kim, "The Modulatory Effect of Acupuncture on the Activity of Locus Coeruleus Neuronal Cells: A Review," Evidence-Based Complementary and Alternative Medicine, vol. 2017, Article ID 9785345, 8 pages, 2017.

[11] National Assembly Research Service, 2017 Policy Report for Inspect of the Government Offices, National Assembly Research Service, Seoul, Republic of Korea, 2017.

[12] J. Yu, C. Kim, K. Kim, J. Lee, and M. Kim, "Behaviors of Providers of Traditional Korean Medicine Therapy and Complementary and Alternative Medicine Therapy for the Treatment of Cancer Patients," Journal of Pharmacopuncture, vol. 18, no. 1, pp. 27-35, 2015.

[13] Hye-Lim Park, Hun-Soo Lee, Byung-Cheul Shin et al., "Traditional Medicine in China, Korea, and Japan: A Brief Introduction and Comparison," Evidence-Based Complementary and Alternative Medicine, vol. 2012, Article ID 429103, 9 pages, 2012.

[14] L. Wang, S. Suo, J. Li et al., "An investigation into traditional chinese medicine hospitals in China: Development trend and medical service innovation," International Journal of Health Policy and Management, vol. 6, no. 1, pp. 19-25, 2017.

[15] Taiwan Medical Association, Notice Revison of Traditional Chinese Medicine Doctor, http://www.tma.tw/meeting/meetinginfo02.asp ?/7645.html.

[16] K. Diaconu, Y. Chen, S. Manaseki-Holland, C. Cummins, and R. Lilford, "Medical device procurement in low- and middleincome settings: protocol for a systematic review," Systematic Reviews, vol. 3, no. 118, pp. 1-11, 2014.

[17] Korea Institute for Advancement of Technology, 2010 Industry Leading Technology Roadmap Executive Summary: Next Generation Medical Devices, Korea Institute for Advancement of Technology, Seoul, Republic of Korea, 2010.

[18] Medical Device Research Division of National Institute of Food and Drug Safety Evaluation, Medical Device Forecast Analysis Report, National Institute of Food and Drug Safety Evaluation, Osong, Republic of Korea, 2017.

[19] J. H. Kim, J. U. Kim, and K. H. Kim, "urvey of Traditional Korean Medical Device: Number of Devices, Problems, Solution Plan," Korean Journal of Oriental Physiology \& Pathology, vol. 28, no. 4, pp. 430-439, 2014.

[20] Y. H. Sakong and B. H. Cho, "A Critical Review of the Court Decisions on the Korean Oriental Doctor's Use of Diagnostic Imaging Devices," Journal of Regulation Studies, vol. 22, no. 2, pp. 225-260, 2013.

[21] Health Network, Chinese and Western medicines open each other, 2017, http://cm.39.net/0711/9/161512.html.

[22] P. Yu, H. Jiang, J. Liu et al., "Traditional Chinese Medicine Treatment for Ruptured Lumbar Disc Herniation: Clinical Observations in 102 Cases," Orthopaedic Surgery, vol. 6, no. 3, pp. 229-235, 2014.

[23] T. Yu, L. M. Xie, B. Wu, Y. B. Li et al., "Research on the Distribution Difference of MRI Signals in Osteonecrosis of the Femoral Head Patients of Different TCM Syndrome Types," Zhongguo Zhong Xi Yi Jie He Za Zhi, vol. 33, no. 12, pp. 1617$1620,2013$. 
[24] Y. Bai, L. Yuan, K. S. Soh et al., "Possible applications for fascial anatomy and fasciaology in traditional Chinese medicine," JAMS Journal of Acupuncture and Meridian Studies, vol. 3, no. 2, pp. 125-132, 2010.

[25] National Low Information Center, Decision $2005 \mathrm{Nu} 1758$ Delivered on June 30. 2006., 2017, http://www.law.go.kr/ precInfoP.do? mode $=0$ \&precSeq $=70463$.

[26] Seoul Administrative Court, Decision 2008 Guhab 11945 Delivered on Oct 10. 2008., 2017, http://sladmin.scourt.go.kr/ dcboard/new/DcNewsViewAction.work? seqnum=4301\&amp; gubun $=44 \&$ amp;scode $\_$kname $=\%$ BF\%EC\%B8\%AE\%B9\%FD $\%$ BF\%F8\%20\%C1\%D6\%BF\%E4\%C6\%C7\%B0\%E1\&amp;currentPage $=57 \&$ amp; searchWord $=\&$ amp;cbub_code $=000220$.

[27] Constitutional Court of Korea, Decision 2010 Hun-ma 109 Delivered on Feb 23. 2012., 2017, http://search.ccourt.go.kr/ ths/pr/selectThsPr0101List.do.

[28] National Law Information Center, Medical Service Law, http:// www.law.go.kr/\%EB\%B2\%95\%EB\%A0\%B9/\%EC\%9D\%98\%EB\% A3\%8C\%EB \%B2\%95.

[29] National Law Information Center, Medical Technicians, http:// www.law.go.kr/\%EB\%B2\%95\%EB\%A0\%B9/\%EC\%9D\%98\%EB\% A3\%8C\%EA \%B8\%B0\%EC\%82\%AC\%EB\%93\%B1\%EC\%97\%90\% EA\%B4\%80\%ED\%95\%9C\%EB \%B2\%95\%EB\%A5\%A0/\%2813367, $20150622 \% 29$.

[30] Constitutional Court of Korea, Decision 2012 Hun-ma 551.561 Delivered on Dec 26. 2013., 2017, http://search.ccourt.go.kr/ths/ pr/ths_pr0103_Print.do?cId=010300\&seq $=0 \&$ cname $=\% \mathrm{ED} \% 8 \mathrm{C} \%$ 90\%EB\%A1\%80\%EC\%A7\%91\&eventNo=2012\%ED\%97\%8C\%EB\% A7\%88551\&pubFlag=0\&eventNum $=35457$ \&selectFont $=$ normal $\&$ showHide $=$.

[31] Ministry of Health and Welfare, Announcement of results of designation of specialized hospital (2018 2020), 2017, http:// www.mohw.go.kr/react/al/sal0101vw.jsp?PAR_MENU_ID=04 \&amp;MENU_ID=040101\&amp;page=1\&amp;CONT_SEQ= 343337.

[32] Jaseng TKM hospital, jaseng's precise diagnosis using diagnostic tools such as X-ray, MRI and CT, 2017, http:// www.jaseng.net/activities/medical-examination/.

[33] K. Lim, B. Shin, I. H. Park, S. Y. Park, and M. Hwang, "Daoyin Exercise Therapy for Low Back Pain : A Systematic Review," Korean Society of Chuna Manual Medicine Spine and Nerves, vol. 13, no. 1, pp. 23-33, 2018. 


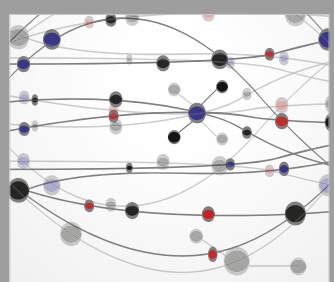

The Scientific World Journal
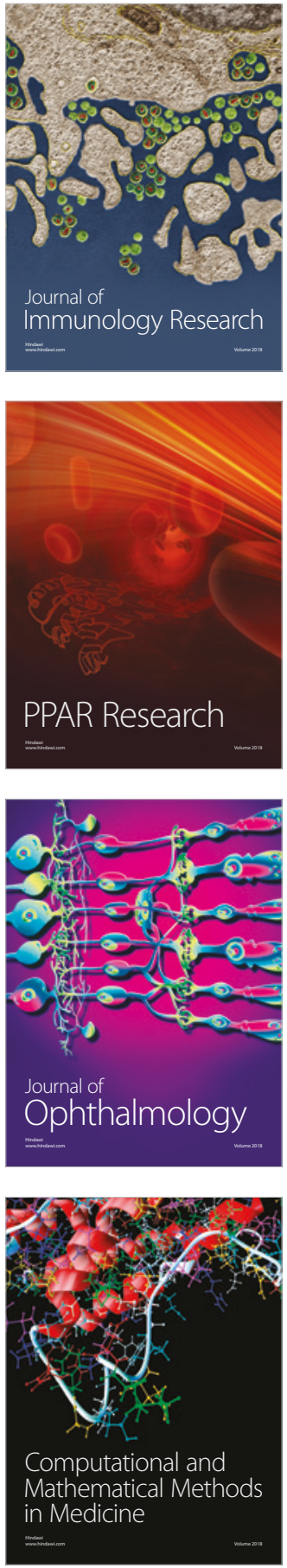

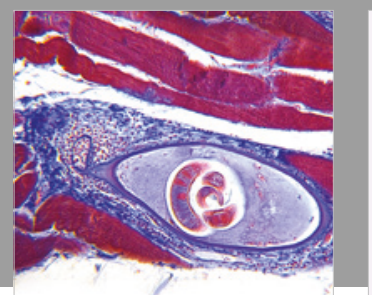

Gastroenterology Research and Practice

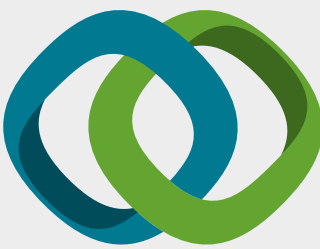

\section{Hindawi}

Submit your manuscripts at

www.hindawi.com
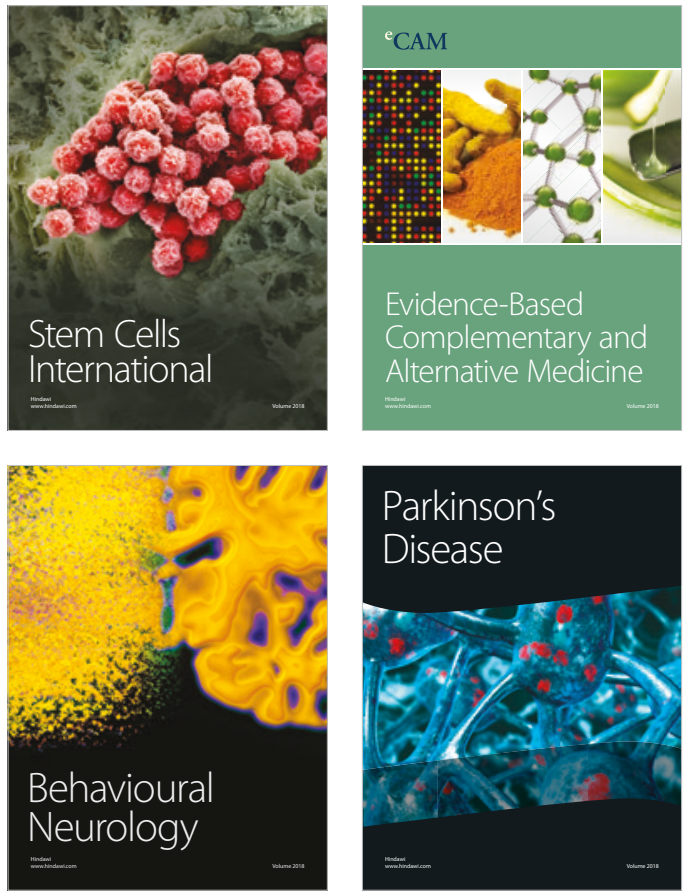

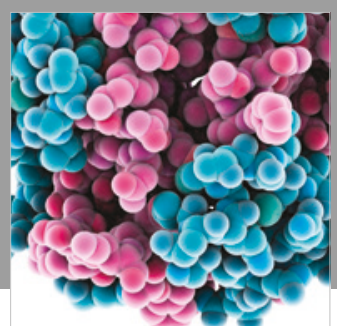

ournal of

Diabetes Research

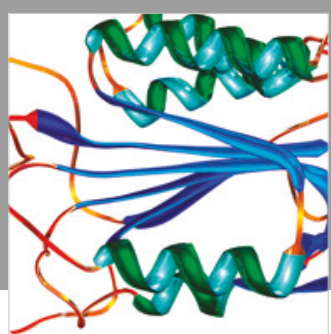

Disease Markers
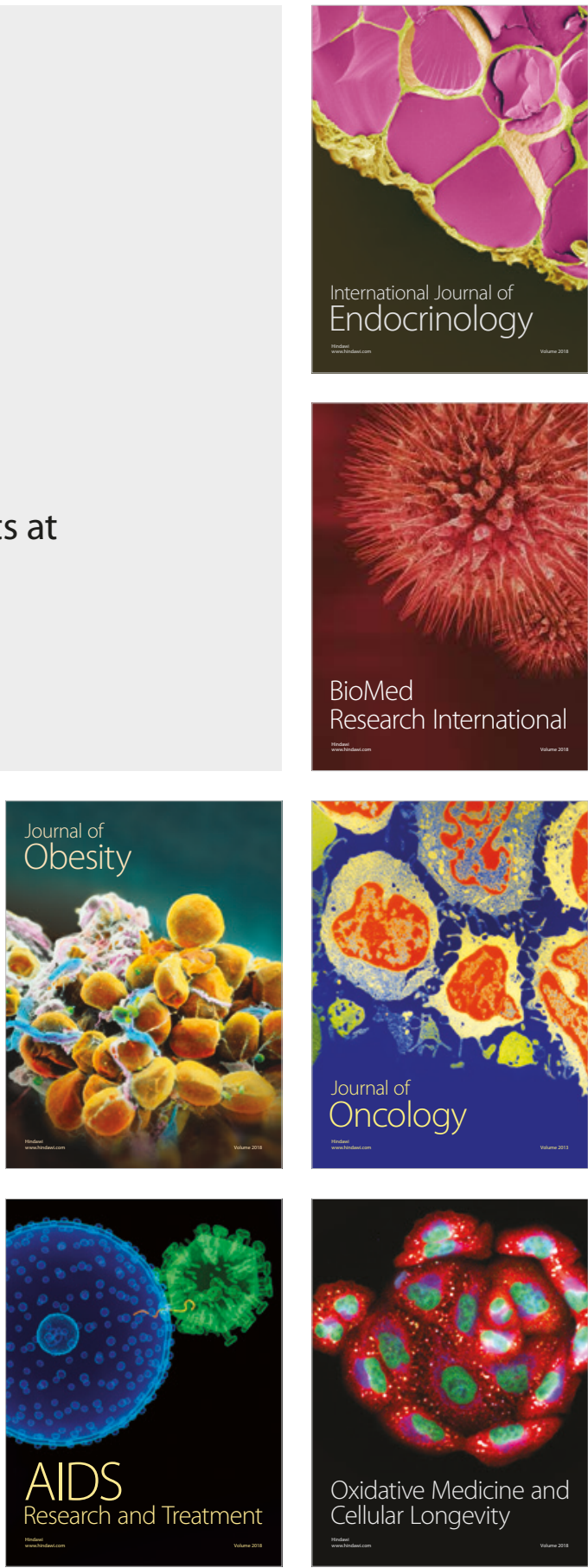